\title{
A study of clinical, histopathological and direct immunofluorescence diagnosis in pemphigus group Utility of direct immunofluorescence
}

\author{
Hrabovska $Z^{1}$, Jautova $\mathbf{J}^{1}$, Hrabovsky $\mathrm{V}^{2}$ \\ Department of Dermatovenerology, Faculty of Medicine, UPJS, Kosice, Slovakia. \\ hrabovska.zu@gmail.com
}

\begin{abstract}
AIMS: To determine the diagnostic accordance between histopathological and direct immunofluorescence diagnosis of patients with autoimmune vesiculobullous skin diseases.

BACKGROUND: The term pemphigus refers to a group of autoimmune blistering diseases mediated by autoantibodies directed against desmoglein proteins. The differentiation between the various bullous diseases is important for treatment and prognosis. Direct immunofluorescence microscopy is still the gold standard in differentiating these diseases.

METHODS: Patients with clinical diagnosis of vesiculobullous dermatitis from pemphigus group were included in the study. We retrospectively analyzed histopathologic and direct immunofluorescence results from skin or mucosal samples over 15-year period.

RESULTS: 81 patients were included. The accordance was good in pemphigus vulgaris and pemphigus herpetiformis, but low in pemphigus vegetans, pemphigus foliaceus and pemphigus erythematosus. No accordance was in Hailey-Hailey disease and Grover's disease. Uncommon result in our analysis included: intraepidermal IgG and IgM depositions at DIF in one Grover's disease patient.

CONCLUSION: Our results confirmed the relevance of direct immunofluorescence assays as a necessary diagnostic method for the definitive diagnosis of autoimmune blistering disease in the case, where the clinical feature and the results of histopathology are not clear (Tab. 4, Fig. 5, Ref. 26). Text in PDF www.elis.sk. KEY WORDS: autoimmnune bullous diseases, histopathology, direct immunofluorescence, pemphigus.
\end{abstract}

\section{Introduction}

Autoimmune bullous diseases include organ-specific diseases that damage the skin and mucous membranes. They are divided into the two main groups, depending on the location of the deposit blister: pemphigus group, where the blister is localized intraepidermally and pemphigoid group, where the blister is localized subepidermally. Differentiate them, as well as differentiation from other bullous diseases, is important for the treatment and prognosis. Among the diseases, the pemphigus group includes pemphigus vulgaris, pemphigus vegetans (Neumann type and Hallopeau type), pemphigus foliaceus, pemphigus erythematosus, pemphigus herpetiformis, IgA pemphigus and paraneoplastic pemphigus. Some of them are potentially fatal diseases of the skin and mucous membrane (1). Autoantibodies directed against desmosomes of epidermal keratinocytes, cadherins, causing loss of adhesion be-

${ }^{1}$ Department of Dermatovenerology, Faculty of Medicine, UPJS, Kosice, Slovakia, and ${ }^{2}$ Department of Medical Microbiology and Clinical Microbiology, Faculty of Medicine, UPJS, Kosice, Slovakia

Address for correspondence: Z. Hrabovska MD, Department of dermatovenerology, Faculty of Medicine, UPJS, Trieda SNP 1, SK-040 01 Kosice, Slovakia.

Phone: +421.911963789 tween keratinocytes, resulting in the formation of large blisters on the skin and mucous membranes. Desmoglein 3 and desmoglein 1, desmosomals glycoproteins, are the target antigens for these diseases (2). Histopathological investigation (HIS) is showing intraepidermal blister, acantholysis a mild inflammatory infiltrate in the superficial parts of the skin. Direct immunofluorescence investigation (DIF) of the skin taken from the closest area of the blister reveals deposits of antibodies (IgG, IgA, IgM, C3) in the intercellular spaces (ICS) of the keratinocytes. For a definitive diagnosis of autoimmune bullous diseases, it is inadequate to realise only histological examination. DIF with using the patient's tissue is the gold standard in the differential diagnosis of these diseases. It is important to use DIF for distinguishing between the pemphigus diseases and Hailey-Hailey disease.

The aim of this study was to analyze the diagnostic accordance between histopathological and DIF diagnosis and to evaluate the significance of the two examinations in the diagnosis of autoimmune vesiculobullous diseases from pemphigus group. Patients were examined at the University of P.J. Šafarik in Košice, Faculty of Medicine, Department of Dermatovenerology between January 1998 and December 2012. Histological processing and examination of tissues with histopathological results was performed at the Department of Pathology, Hospital University of L. Pasteur in Košice. 


\section{Material and methods}

Totally, 81 cases with clinical diagnosis as vesiculobullous dermatitis that had been diagnosed in our department between January 1998 and December 2012, were included in our study. Hailey-Hailey disease cases were included in our study because Hailey-Hailey disease is important in the differential diagnosis of pemphigus group. We retrospectively analyzed the histopathological and immunofluorescence records of the patients diagnosed with autoimmune bullous dermatoses. The inclusion criteria were: a histopathologic exam suggesting bullous dermatosis and a simultaneous DIF of the samples of the skin and mucous membranes during admission. Diseases belonged to the group of intraepidermal blistering diseases (pemphigus vulgaris - PV, pemphigus vegetans - PVeg, pemphigus foliaceus - PF, pemphigus erythematosus $\mathrm{PE}$, pemphigus herpetiformis - $\mathrm{PH}$, Hailey-Hailey disease $-\mathrm{HH}$ and Grover's disease - GDT. Tissue samples of skin or mucous membranes of patients with autoimmune bullous disease were used for the study.

\section{Histological examination}

The histological examination of biopsy was collected from the skin, containing a fresh blister (vesicle and bulla), lasting less than 24 hours. Excised was completely and subsequently saved in formaldehyde. In the case of pathological findings in the mucosa, biopsied material was taken from erosion because blisters were still very short. Tissue samples were examined by histopathology from the tissue blocks in formalin-fixed and paraffin-embedded. After histological processing, the preparation was stained with Mayer's hematoxylin and eosin.

Positivity of the samples was determined by finding and localization of the blister having underlying acantholysis intraepidermally. PV was confirmed by suprabasal blister, acantholytic cells in the epidermis and in view of "advice tombstones" in the stratum basale. PVeg was confirmed by suprabasal acantholytic blistering massive acanthosis, hyperkeratosis and papillomatosis. In the Hallopeau type, there were intraepidermal microabscesses with eosinophilic leukocytes, in the Neumann type typically intraepidermal vesicles. PF was confirmed by histological changes with blistering and subcorneal acantholysis in stratum spinosum and stratum granulosum and dermal perivascular lymphocytic infiltrate. PE was confirmed by subcorneal localized blister, acantholytic cells and basement membrane thickening. PH was positive with superficial acantholytic blisters, or eosinophilic spongiosis. IgA pemphigus has an eosinophilic spongiosis in a histological feature. HH was positive with acantholysis, with slightly acanthosis of epidermis forming "collapsed brick wall", hyperplasia and parakeratosis. In GD were positive changes in the form of the epidermal acantholysis with suprabasal or subcorneal creating splits and spongiosis. Samples referred to as indeterminate did not present the typical histological features of the disease.

\section{Immunofluorescence examination}

For a direct immunofluorescence, examination was taken from patient biopsy sample of the skin or mucosa from the edge of the blister resp. erosion. The sample was quickly frozen at $-20{ }^{\circ} \mathrm{C}$. Frozen tissue was sectioned on a cryostat to a thickness of 6-8 microns and placed on adhesive slides. Prepared glasses were marked by antibody (Ig, IgG, IgA, IgM, C3) and by the identification number of the patient. After a short drying, the glass at room temperature was washed with phosphate buffer (PBS), $\mathrm{pH}$ 7.2-7.4; three times for $5 \mathrm{~min}$. Next, the tissue sections were directly labelled by immunofluorescent antibody diluted as recommended by the manufacturer, in order to optimize the laboratory (1:6). Incubation with the antibodies was carried out in the dark, at room temperature in a humid chamber for 30 minutes. After incubation, the solution remained sintered glass and drying them. Subsequently, the glass was again thoroughly rinsed with phosphate buffered saline, blotted and mounted in glycerine mounting medium. Thus, prepared formulations were evaluated for fluorescent microscope BX51, Olympus.

For direct immunofluorescence tests, immunoconjugates by manufacturer Nordic Immunology (NE) were used: SwAHu/Ig FITC, SwAHu/IgG FITC, SwAHu/IgA FITC, SwAHu/IgM FITC, $\mathrm{SwAHu} / \mathrm{C} 3 \mathrm{c}$ FITC.

DIF in PV were positive at the network-finding deposits of immunoglobulin (IgG, IgA) and complement (C3) between the cells of the epidermis, affecting all layers of the epidermis. In PVeg and PF a net-positive deposits of the same immunoglobulin (IgG) and complement (C3) intercellular, intraepidermally, in PF localized predominantly subcorneal. The DIF was positive in PE with the finding of intercellular deposition of immunoglobulin (IgG, IgM), and complement $(\mathrm{C} 3)$ between the cells of the epidermis, which primarily affected subcorneal layer of the epidermis and at the same time homogeneous deposits of $\mathrm{IgG} / \mathrm{C} 3$ along the basement membrane zone. In PH, we observed positive findings of networklike deposits of immunoglobulin (IgG, IgA) and complement (C3) between the cells of the epidermis, affecting all layers of the epidermis. IgA pemphigus was present only by positivity of DIF IgA antibodies in the intercellular spaces of the epidermis. In $\mathrm{HH}$, the finding of immunoglobulins and complement in direct immunofluorescence is negative. In GD, imunodeposits of IgG and IgM are saved intraepidermally non-specific and non-homogeneous, event. negative.

Data was analyzed using the Scientific Package for Social Sciences software (SPSS).

Tab. 1. Diagnosis in numbers and percentages.

\begin{tabular}{lcc}
\hline Diagnosis & $\begin{array}{c}\text { Number } \\
\text { of cases (n) }\end{array}$ & $\begin{array}{c}\text { Percentages } \\
\text { of cases (\%) }\end{array}$ \\
\hline Pemphigus vulgaris & 52 & 64.2 \\
Pemphigus vegetans & 5 & 6.2 \\
Pemphigus foliaceus & 5 & 6.2 \\
Pemphigus erythematosus & 7 & 8.7 \\
Pemphigus herpetiformis & 5 & 6.2 \\
Hailey-Hailey disease & 5 & 6.2 \\
Grover's disease & 2 & 2.5 \\
\hline Total & 81 & 100 \\
\hline
\end{tabular}




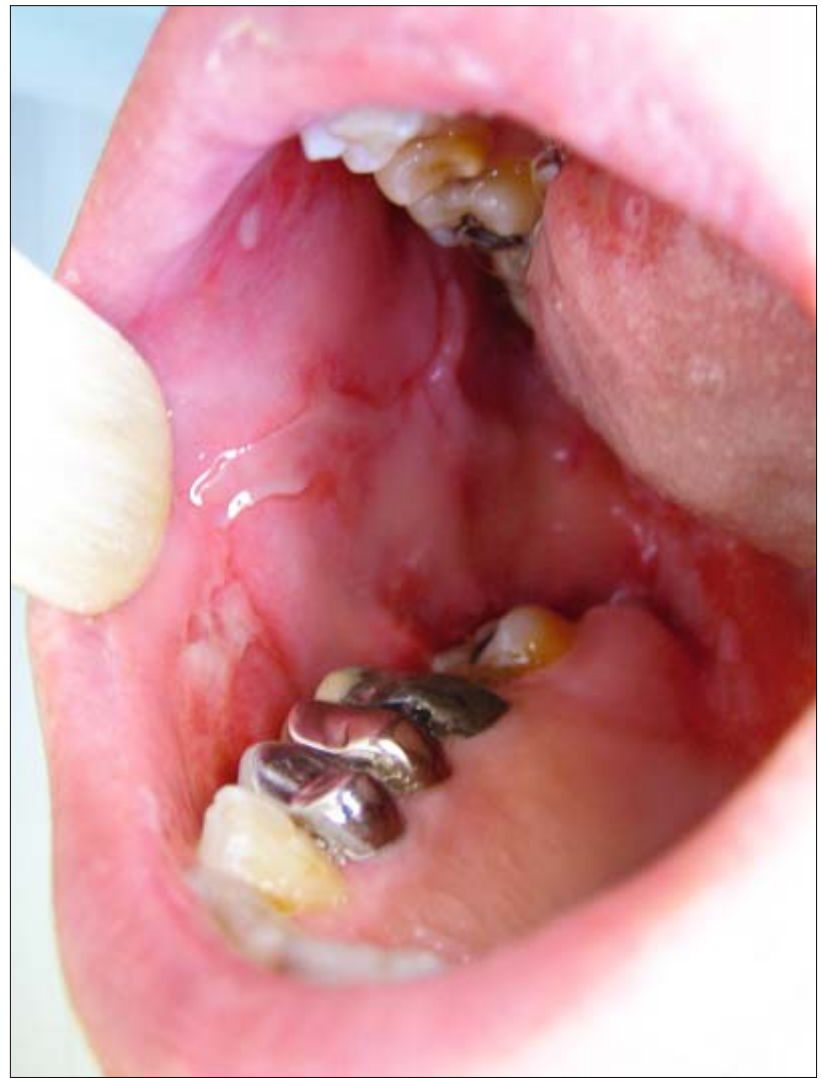

Fig. 1. Pemphigus vulgaris - clinical features of erosions in oral cavity, buccal mucosa.

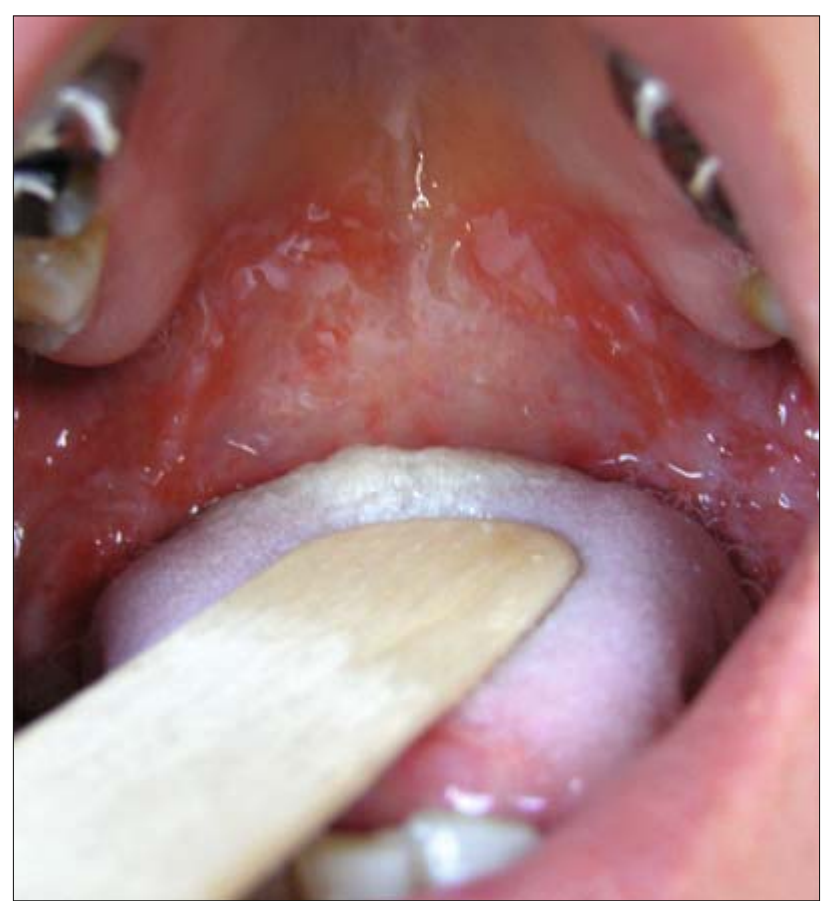

Fig. 2. Pemphigus vulgaris - clinical features of erosions in oral cavity, palate.

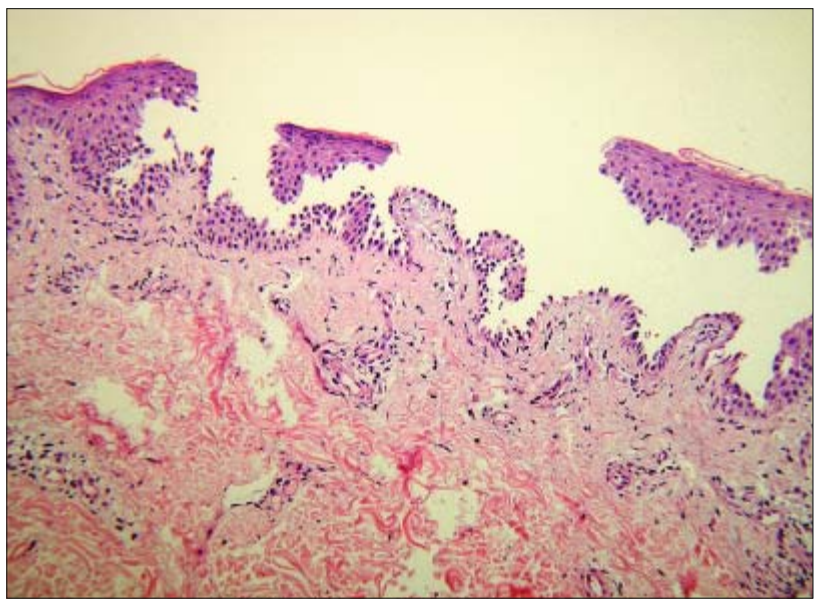

Fig. 3. Histologic findings of pemphigus vulgaris. Intraepidermal blister located above the stratum basale, which is preserved as a "series of tombstones", acantholytic cells in the blister cavity. HE, x100-200.

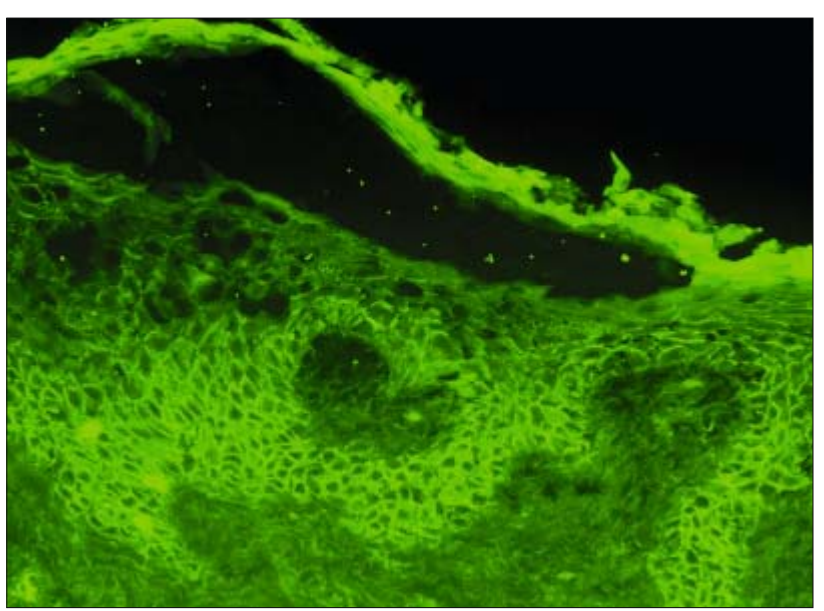

Fig. 4. Direct immunofluorescence of pemphigus vulgaris. Vesicles are intraepidermal, in the intercellular junctions are reticular deposits of IgG in the range of almost all of the epidermis. DIF of IgG, $\mathbf{x 2 5}$.



Fig. 5. Direct immunofluorescence of pemphigus vulgaris. Vesicles are intraepidermal, in the intercellular junctions are reticular deposits of C3. DIF of C3, $\mathrm{x} 25$ 
Tab. 2. Histopathological results in pemphigus group.

\begin{tabular}{lccc}
\hline Diagnosis & Confirmed $(\%)$ & Non-Confirmed $(\%)$ & 0 \\
\hline Pemphigus vulgaris & $47(90.4)$ & 0 & Uncertain $(\%)$ \\
Pemphigus vegetans & $4(80.0)$ & $1(20.0)$ & $1(20.0)$ \\
Pemphigus foliaceus & $2(40.0)$ & $1(14.3)$ & 0 \\
Pemphigus erythematosus & $3(42.9)$ & 0 & $3(40.0)$ \\
Pemphigus herpetiformis & $4(80.0)$ & 0 & 0 \\
Hailey-Hailey disease & $5(100.0)$ & 0 \\
Grover's disease & $2(100.0)$ & $0.0)$ \\
\hline
\end{tabular}

Tab. 3. Antibody depositions in direct immunofluorescence.

\begin{tabular}{|c|c|c|c|c|c|}
\hline Diagnosis & $\operatorname{IgA}(\%)$ & $\operatorname{IgG}(\%)$ & $\operatorname{IgM}(\%)$ & $\mathrm{C} 3(\%)$ & Negative $(\%)$ \\
\hline Pemphigus vulgaris & $1(2.2)$ & $42(91.3)$ & 0 & $42(91.3)$ & $4(8.7)$ \\
\hline Pemphigus vegetans & 0 & $4(100.0)$ & 0 & $2(50.0)$ & 0 \\
\hline Pemphigus foliaceus & 0 & $5(100.0)$ & 0 & $3(60.0)$ & 0 \\
\hline Pemphigus erythematosus & 0 & $6(100.0)$ & $1 \quad(16.7)$ & $6(100.0)$ & 0 \\
\hline Pemphigus herpetiformis & $2(40.0)$ & $4(80.0)$ & 0 & $4(80.0)$ & $1(20.0)$ \\
\hline Hailey-Hailey disease & 0 & 0 & 0 & 0 & $5(100.0)$ \\
\hline Grover's disease & 0 & $1(50.0)$ & $1 \quad(50.0)$ & 0 & $1(50.0)$ \\
\hline
\end{tabular}

Tab. 4. Comparison between positive histopathological and direct immunofluorescence findings.

\begin{tabular}{|c|c|c|c|c|}
\hline \multirow[b]{2}{*}{ DIAGNOSIS } & \multicolumn{2}{|c|}{ HIS } & \multicolumn{2}{|c|}{ DIF } \\
\hline & positive $(\%)$ & $\begin{array}{l}\text { investigations }(\mathrm{n}) / \\
\text { patients }(\mathrm{n})\end{array}$ & positive $(\%)$ & $\begin{array}{c}\text { investigations }(\mathrm{n}) / \\
\text { patients }(\mathrm{n})\end{array}$ \\
\hline Pemphigus vulgaris & $47(90.4)$ & $52 / 52$ & $42(91.3)$ & $46 / 52$ \\
\hline Pemphigus vegetans & $4(80.0)$ & $5 / 5$ & $4(100)$ & $4 / 5$ \\
\hline Pemphigus foliaceus & $2(40.0)$ & $5 / 5$ & $5(100)$ & $5 / 5$ \\
\hline Pemphigus erythematosus & $3(42.9)$ & $7 / 7$ & $6(85.7)$ & $7 / 7$ \\
\hline Pemphigus herpetiformis & $4(80.0)$ & $5 / 5$ & $4(80)$ & $5 / 5$ \\
\hline Hailey-Hailey disease & $5(100)$ & $5 / 5$ & 0 & $5 / 5$ \\
\hline Grover's disease & $2(100)$ & $2 / 2$ & 0 & $2 / 2$ \\
\hline
\end{tabular}

Grover's disease $2(100)$

$2 / 2$

\section{Results}

During the 15-year period, 81 newly diagnosed cases of pemphigus occurred in the district of Košice in Slovakia (Tab. 1).

Pemphigus vulgaris was the most common clinical variant, diagnosed in $52(64.2 \%)$ patients, followed by pemphigus erythematosus in 7 patients $(8.7 \%$ ), pemphigus vegetans, pemphigus foliaceus, pemphigus herpetiformis and Hailey-Hailey disease in 5 patients $(6.2 \%)$; and Grover's disease in 2 patients $(2.5 \%)$. None of the IgA pemphigus or paraneoplastic pemphigus has been detected.

The number of women with PV was in the majority compared to men, the female to male ratio was $1.6: 1$. The mean age at the onset of the disease was 51 years. In the group of patients with $\mathrm{PE}$, in two patients was a positive family history with systemic autoimmune diseases, specifically with rheumatoid arthritis and vasculitis.

Clinical manifestations: in all patients, we analyzed the localization of the clinical symptoms on the skin and mucous membranes. In pemphigus vulgaris, it was in $34.6 \%$ manifested by skin type without mucosal involvement, in $17.3 \%$ by mucosal type (Figs 1 and 2) and in $48.1 \%$ of the cases, the patients had mixed type of the disease. In the case of pemphigus vegetans, we observed in $60 \%$ of the cases symptoms simultaneously on the skin and mucosa, in $40 \%$ only exclusively cutaneous form of the disease. In the variant of pemphigus foliaceus, in $80 \%$ of the cases, the patients were only with skin involvement and in $20 \%$, with the symptoms of the mucous membranes. In patients with a diagnosis of pemphigus erythematosus, $85.7 \%$ of the cases had skin changes only, and $14.3 \%$ were reflected on the mucosa, particularly in the oral cavity. All five patients with pemphigus herpetiformis had pathological changes on the skin only, without mucosal involvement. In Hailey-Hailey disease, $80 \%$ of the cases had only a lesion on the skin, and $20 \%$ on the genital mucosa. Two patients with Grover's disease were described with a skin changes. After the start of immunosuppressive treatment using methylprednisolone, relieving clinical symptoms occurred in most patients after a few weeks. In other cases, where there had not been clinical remission, it was necessary to add the adjuvant treatment of immunosuppressive drugs as azathioprine ev. cyclophosphamide.

Histopathological results of all diseases are shown in the Table 2. Histopathological diagnosis was based on the level of blister separation and the presence of acantholytic cells. PV was confirmed by histopathological examination in $90.4 \%(47 / 52)$ (Fig. 3). In $9.6 \%(5 / 52)$, the results were uncertain. PVeg was confirmed in $80 \%(4 / 5)$, in $20 \%(1 / 5)$ the result was uncertain. PF was confirmed in $40 \%(2 / 5)$, non-confirmed in $20 \%(1 / 5)$ and uncertain in $40 \%(2 / 5)$. PE was confirmed in $42.9 \%(3 / 7)$, nonconfirmed in $14.3 \%(1 / 7)$ and uncertain in $42.9 \%(3 / 7)$. PH was confirmed in $80 \%(4 / 5)$, uncertain in one case. All patients with 
HH had typical intraepidermal changes. In two patients with GD, epidermal changes were observed, concretely irregular acanthosis, focal parakeratosis, dyskeratosis, spongiosis and acantholysis with suprabasal blister.

In all cases, where the result of histological examination was uncertain, it was necessary to realize the immunofluorescence examination simultaneously and compare the results with the clinical picture.

As displayed in the Table 3, the positive DIF findings were as follows: PV $91.3 \%$ (42/46): intercellular, intraepidermal IgG: $91.3 \%$ (42/46) (Fig. 4); intercellular, intraepidermal C3 in 91.3 $\%(42 / 46)$ (Fig. 5) and intercellular, intraepidermal IgA in $2.2 \%$ (1/42). In 4 cases was DIF negative. PVeg $100 \%$ (4/4): intercellular, intraepidermal IgG in $100 \%$ (4/4); intercellular, intraepidermal $\mathrm{C} 3$ in $50 \%(2 / 4)$. PF $100 \%(5 / 5)$ : intercellular, intraepidermal IgG in $100 \%(5 / 5)$; intercellular, intraepidermal C3 in $60 \%(3 / 5)$. PE $85.7 \%$ (6/7): intercellular, intraepidermal IgG in $100 \%(6 / 6)$; intercellular, intraepidermal C3 in $100 \%(6 / 6)$ and intercellular, intraepidermal $\operatorname{IgM}$ in $16.7 \%$ (1/6). In one case was DIF uncertain. Homogenous deposits of $\mathrm{IgG} / \mathrm{C} 3$ at the dermoepidermal junction were also found in 5 cases and circulating antinuclear antibodies in serum in four cases. PH $80 \%$ (4/5): intercellular, intraepidermal IgG in $80 \%(4 / 5)$; intercellular, intraepidermal C3 in $80 \%$ (4/5); intercellular, intraepidermal IgA in $40 \%(2 / 5)$. In one case was DIF negative. HH $0 \%(0 / 5)$ : no antibodies found. GD $0 \%$ $(0 / 2)$ : immunodeposits of $\operatorname{IgG}$ and $\operatorname{IgM}$ were nonspecific in one case, located intraepidermal and nonhomogenous, in one case was DIF negative.

The aim of this study was to evaluate accordance between histological and immunofluorescence diagnosis in pemphigus group and to analyze the importance of both individual examinations in the tissue diagnosis of diseases (Tab. 4). From the 52 patients with $\mathrm{PV}$, all patients had realized a histopathology exam, positive result had $90.4 \%$ of the patients. On the other hand, DIF examination was realized only in 46 patients, but positive results were in 91.3 $\%$. Positivity for both examinations was above $90 \%$. Based on this result, we could consider both examinations as equivalent in the diagnosis of PV.

All 5 patients with PVeg had realized histology with positivity in $80 \%$. DIF testing was realized only in 4 patients, with positive results in $100 \%$. In this condition, the immunofluorescence features are the same as PV. In this case, the correlation of clinical signs with the results of investigation methods is necessary. In other diseases, all patients made both tests and the results are as followed: PF: histopathology exam positive in $40 \%$, DIF testing positive in $100 \%$; PE: histopathology exam positive in $42.9 \%$, DIF testing positive in $85.7 \%$. The diagnosis of these diseases was significantly more specific by DIF and considered it in these cases as necessary examination. $\mathrm{PH}$ had in both cases positive results in $80 \%$, so we could consider in this diagnosis both examinations as equivalent. HH disease: positive histopathology in $100 \%$, DIF negative in all cases. Whereas, the histopathological features are the same as in PV, negative DIF exclude PV and plays a key role in the diagnosis of this disease. GD: histological examination was specific, whereas DIF was negative, resp. nonspecific.

\section{Discussion}

Various primary skin diseases are clinically presented as vesiculobullous lesions, but their aetiology, pathogenesis, severity and clinical course are different. It is therefore necessary to accurately diagnose the diseases, and also the subsequent treatment with a suitable conducting to prevent or minimize the risk of associated morbidity and mortality. In recent years, diseases were the subject of intense research.

In our study, the most common was PV in $64.2 \%$, followed $\mathrm{PE}$ in $8.7 \%$, PVeg in $6.2 \%$, PF in $6.2 \%$, PH in $6.2 \%$, HH in 6.2 $\%$ and GD in $2.5 \%$. None of the IgA pemphigus or paraneoplastic pemphigus has been detected. PV was found to outnumber other types of pemphigus in India (3), Korea (4), Iran (5), Macedonia (6), Croatia (7) and in Spain (8), in contrast to the higher prevalence of PE in Finland (9). The notable exceptions were Brazil and other Latin American countries in which fogo selvagem, an endemic form of PF, predominated (10).

The number of women with PV was in the majority compared to men, the female to male ratio was $1.6: 1$, which agrees with the data of most previous studies worldwide. The mean age at the onset of the disease was higher in our patients, when compared to the study from Pakistan (11) and Iran (5). It was nearly the same in some other countries, e.g. Croatia (12) and also in our country from another study of PV (13).

Not all patients with autoimmune bullous diseases have the typical clinical signs and distribution of lesions on the skin and mucous membranes. The differences may be due to the severity and stage of disease at the time of examination, but also due to the previous treatment. The typical feature of the pemphigus is primary involvement of the oral mucosa (1), as the proportion of patients in our group. In cases where the clinical diagnosis is difficult, histopathological examination and direct immunofluorescence of biopsy specimens of skin or mucosa will help to determine the final diagnosis.

All vesiculobullous diseases showed specific histopathological changes that are apparent only when the biopsy sample was taken from an intact vesicle or bulla. Several studies have evaluated various specific histological findings of the location of blisters, epidermal changes, e.g.: acantholysis, hyperkeratosis, acanthosis, dyskeratosis, or the presence of inflammatory infiltrate etc. $(14,15)$. Similar to the clinical manifestations, histopathological changes may be different. In our study, we aimed, by the evaluation of histopathological results, if the histopathological specimen confirms the diagnosis of the autoimmune vesiculobullous disease, or not. It was established on the basis of clearly defined histopathological features. In cases where nonspecific changes were present, and the result was inconclusive, the sample was evaluated as uncertain.

PV was confirmed in $90.4 \%$ of the cases, whereas, $9.6 \%$ of the cases were indeterminate. PVeg was confirmed in $80 \%$ of the cases and in $20 \%$ the results were uncertain. PF was confirmed in $40 \%$ of the cases, in $20 \%$ non-confirmed and in $40 \%$ the results were uncertain. PE was confirmed in $42.9 \%$ of the cases, non-confirmed in $14.3 \%$ and uncertain in $42.9 \%$. PH was in $80 \%$ clearly confirmed, in $20 \%$ the results were uncertain. HH and GD were 
in all cases confirmed by histological examination. Ljubojević et al confirmed by histological examination diagnosis of the PV in $98 \%$ of the cases (12). If histopathological examination did not confirm the disease and clinical manifestations were not clear, examination by DIF was necessary in these cases.

The most important techniques in the investigation of patients with vesiculobullous disease in addition to conventional histopathology includes confirmatory tests such as: direct and indirect immunofluorescence (16). These immunofluorescent methods are fast and reliable. They can be used to establish early diagnosis and subsequent treatment of these potentially life-threatening diseases (17). Direct immunofluorescence of perilesional skin and mucous membranes detects intercellular deposits of immunoreagents. This is a sensitive and specific method. For the detection of serum antibodies, an indirect immunofluorescence technique can be used. Indirect immunofluorescence using antibodies binding to antigens of the patient in a substrate of the human skin or other sensitive substrate. It evaluates quantitative antibody levels; antibody titre correlates with the severity of the disease (1). It may be negative at the beginning of the disease when the disease is localized only on the mucosa. For detection of the antibodies directed to desmoglein, this method can not distinguish antibody binding to the various types of desmoglein, and also differentiate the different types of pemphigus. Immunoblotting and ELISA use recombinant antigens. Immunoblotting is an effective method, but it is complicated, time consuming and unsuitable for routine practice. ELISA uses recombinant proteins, representing epitopes of natural antigens Dsg1 and Dsg3. The method is highly sensitive and specific. As indirect method is more suitable for determining the activity of the disease and to assess the success of treatment (18).

Direct immunofluorescence method facilitates a detection of molecules such as: immunoglobulins and complement using a biopsy sample $(19,20)$. Only this way can the attached autoimmune reactants in the tissue directly demonstrated. That is why this method is crucial in the diagnosis of autoimmune intraepidermal and subepidermal bullous diseases. Chams-Davatchi et al described positive DIF in $93.28 \%$ of cases of PV (5), Deepti et al in $94.11 \%$ of cases (17) and Kanwar et al up to $100 \%$ of the cases (21). In our study, DIF was positive in $91.3 \%$ of samples.

In PVeg, all our patients had a positive DIF. In the diagnosis of PVeg, the clinical picture and histopathological result play an important role. DIF is important in differentiation from other diseases, such as Darier's disease and Hailey-Hailey disease, in which the DIF is negative.

In cases of PF, our results were the same as in the group of patients of Inchara et al (22) and Deepti et al (17). All testing samples of the patients were positive by the DIF. Chams-Davatchi et al described positive DIF in $88 \%$ of cases (5). If the findings of the DIF in pemphigus foliaceus are similar to pemphigus vulgaris, histopathological examination will help in differentiating these two diseases. In this case, DIF is only the supplementary examination, and is not a substitute for histological examination.

In PE, they were positive in $85.7 \%$ of the cases, $14.3 \%$ of the samples had uncertain results. DIF results were positive in more cases than in histology investigation. PE and PF have similar his- topahological findings, but in direct immunofluorescence, pemphigus erythematosus usually up to $80 \%$ showed granular deposits along the basement membrane zone with intercellular deposits in the epidermis. This unite is associated with immunopathological process of pemphigus foliaceus and lupus erythematosus. In the case of uncertain results, the final diagnosis was established on the basis of circulating antinuclear antibodies in the blood. In PH, positive samples were in $80 \%$, negative in $20 \%$. Negative DIF in all cases of $\mathrm{HH}$ confirmed this diagnosis.

One patient with GD had a negative DIF and one patient had non-specific result of DIF.

Uncommon result in our study included: intraepidermal IgG and IgM depositions at DIF in one Grover's disease patient, suggesting a possible complementary role for autoimmunity in the pathogenesis in this condition (23). Diffuse granular deposits of IgM, C3 and fibrin may occur in the basement membrane zone as the result of vessel wall injury. Of course, caution is needed in the evaluation of clinical and pathological significant immunofluorescent signs (24).

In 4 cases with clinical and histological features of PV, the result of DIF was negative.

DIF is performed using the perilesional skin that is normal appearing skin immediately adjacent to a lesion. The immunodeposits are partially or completely degraded in inflamed or blistered skin and DIF may be negative. Correctly taken biopsy plays an essential role to achieve the best sensitivity of DIF.

Selection of biopsy site, treatment status, and technical errors may result in false negativity of DIF. In the absence of these factors, the negative DIF indicates a prolonged remission (14). Similarly, negative DIF helps in differentiating Hailey-Hailey disease from intraepidermal immunobullous diseases.

In the present study, false-negativity of direct immunofluorescence in PV was due to incorrect selection of the biopsy site or sampling error and sample processing. The study included only patients, who have not yet started immunosuppressive therapy. In one case of GD, autoantibodies of IgG and IgM were presented intraepidermally. This finding was not specific for pemphigus pattern and was leading to the diagnosis of the transient acantholytic dermatosis, based on clinical picture and histological result of the disease.

The differential diagnosis of a DIF test depends on the primary site of immune deposition, the class of immunoglobulin or other type of immune deposits, number of immune deposits and if multiple, the identity of the most intense deposits and deposition in other sites besides the main site. With these parameters, a pattern approach can lead to an accurate diagnosis in the majority of specimens (25). In the case, that DIF is done during the treatment by immunosupresives, the results of DIF can be negative. Negative immunofluorescence may be viewed as the state of immunological remission in pemphigus (26).

DIF is a very reliable diagnostic test for pemphigus group. It becomes positive at a very early stage and remains positive for a long period after clinical remission.

Retrospective analysis and the relatively low number of patients with Grover's disease ( 2 patients) are the main limitations 
of this work. More appropriate would be in the group of autoimmune bullous diseases prospective study using tissue and serological diagnostics.

\section{Conclusion}

Evaluating and comparing the results in our retrospective study, we confirmed the diagnostic significance and consistency in the level of tissue diagnosis with histopathological and direct immunofluorescence investigations in pemphigus vulgaris and pemphigus herpetiformis. In the cases of pemphigus vegetans and pemphigus foliaceus, a direct immunofluorescence was positive in all examined patients, in pemphigus erythematosus in $85.7 \%$ of samples, while histopathological examination was positive in $80 \%, 40 \%$, respectively in $42.9 \%$ of cases. In Hailey-Hailey disease and morbus Grover, diagnosis was established based on histopathological examination, while a direct immunofluorescence eliminated the disease from the group of autoimmune bullous dermatosis.

Despite the more recent sophisticated assay, such as ELISA, immunoblotting, immunoprecipitation, or immunoelectron microscopy, diagnosis of autoimmune bullous dermatoses is still based on a tissue diagnosis and indirect immunofluorescence in many laboratories. In our study, we have seen the importance in the final diagnosis, proper sampling of biopsy and appropriate processing of the sample for histopathology and direct immunofluorescence. The clinical and laboratory correlation is important as well.

The study shows that tissue diagnosis, a combination of investigations of native tissue by a direct immunofluorescence and histological examination, remains the gold standard in the diagnosis of autoimmune bullous dermatosis. Routinely carrying out the indirect immunofluorescence combined with ELISA assay using multiple recombinant antigens, will enhance the sensitivity of the immunoserologic diagnosis in these very similar diseases.

\section{References}

1. Švecová D. Autoimunitné choroby kože II. Autoimunitné bulózne dermatózy. Dematol Prax 2012; 6 (2): 63-67.

2. Švecová D. Pemphigus. Bratislava: Bonus; 2009.

3. Kumar KA. Incidence of pemphigus in Thrissur district, south India. Indian J Dermatol Venereol Leprol 2008; 74: 349-351.

4. Seo PG, Choi WW, Chung JH. Pemphigus in Korea: clinical manifestations and treatment protocol. J Dermatol 2003; 30: 782-788.

5. Chams-Davatchi C, Valikhani M, Daneshpazhooh M et al. Pemphigus: analysis of 1209 cases. Int J Dermatol 2005; 44: 470-476.

6. Vlckova-Laskoska MT, Laskoski DS, Kamberova S et al. Epidemiology of pemphigus in Macedonia: a 15-year retrospective study (19902004). Int J Dermatol 2007; 46: 253-258.

7. Sustic N, Rucevic I, Barisic-Drusko V. Epidemiology of acquired bullous diseases in Eastern Croatia: a retrospective prewar to postwar study. Acta Dermatovenerol Croat 2005; 13: 228-232.
8. Coronel-Pérez IM, Rodríguez-Rey EM, Pérez-Bernal AM, Camacho FM. Epidemiology of pemphigus in the Hospital Universitario Virgen Macarena, Seville, Spain, 2005-2006. Actas Dermosifiliogr 2009; 100: $121-125$.

9. Hietanen J, Salo OP. Pemphigus: an epidemiological study of patients treated in Finnish hospitals between 1969 and 1978. Acta Derm Venereol 1982; 62: 491-496.

10. Abrèu-Velez AM, Hashimoto T, Bollag WB et al. A unique form of endemic pemphigus in northern Colombia. J Am Acad Dermatol 2003; 49: 599-608.

11. Rizvi SR, Sadiq S. Use of direct immunofluorescent microscopy in the diagnosis of vesiculobullous disorders of skin. Pak J Med Sci 2010; 26 (2): 411-415.

12. Ljubojevič $\mathbf{S}$, Lipozenčič $\mathbf{J}$, Brenner $\mathrm{S}$, Budimčič $\mathrm{D}$. Pemphigus vulgaris: a review of treatment over a 19-year period, European Academy of Dermatology and Venerology JEADV 2002;16: 599-603.

13. Chmúrová N, Švecova D. Pemphigus vulgaris: a 11-year review. Bratisl Med J 2009; 110 (8): 500-503.

14. Arundhathi S, Ragunatha S, Mahadeva KC. A Cross-sectional Study of Clinical, Histopathological and Direct Immunofluorescence Spectrum of Vesiculobullous Disorders. J Clin Diag Res 2013; 7: 2788-2792.

15. Leena, Vijaya B, Manjunath GV, Sunila. A Clinico-pathological Study Of 22 Cases Of Pemphigus. J Clin Diag Res 2010; 4:2446-2451.

16. Wojnarowska F, Venning VA. Immunobullous diseases. In: Burns T, Brethnach S, Cox N, Griffiths C. Rook's Textbook of Dermatology, 8th edition. Oxford: Wells Blackwell, 2010, 40.1-40.62.

17. Deepti SP, Sulakshana MS, Manjunatha YA, Jayaprakash HT. A histomorphological study of bullous lesions of skin with special reference to immunofluorescence. Int J Curr Res Aca Rev 2015; 3 (3): 29-51.

18. Yang B, Wang $\mathbf{C}$, Chen $\mathbf{S}$ et al. Accuracy of indirect immunofluorescence on sodium chloride-split skin in the differential diagnosis of bullous pemphigoid and epidermolysis bullosa acquisita. Indian J Dermatol Venereol Leprol 2011; 77: 677-82.

19. Minz RW, Chhabra S, Singh S et al. Direct immunofluorescence of skin biopsy: Perspective of an immunopathologist. Indian J Dermatol Venereol Leprol 2010; 76: 150-157.

20. Jautová J. Imunohistologická diagnostika v dermatógii. Lékařské listy: príloha zdravotníckych novin 2007; 11: 16.

21. Kanwar AJ, De D. Pemphigus in India. Indian J Dermatol Venereol Leprol 2011; 77: 439-449.

22. Inchara YK, Rajalakshmi T. Direct immunofluorescence in cutaneous vesiculobullous lesions. Indian J Pathol Microbiol 2007; 50: 730-732.

23. Millns JL, Doyle JA, Muller SA. Positive cutaneous immunofluorescence in Grover's disease. Arch Dermatol 1980; 116: 515.

24. Valenzuela R, Bergfeld WF, Deodhar SD. Interpretation of immunofluorescent patterns in skin diseases. Chicago: Am Soc of Clin Pathol Press, 1984; 122.

25. Mutasim DF, Adams BB. Immunofluorescence in dermatology. J Am Acad Dermatol 2001; 45: 803-822.

26. Buch AC, Kumar H, Panicker NK et al. A cross-sectional study of direct immunofluorescence in the diagnosis of immunobullous dermatoses. Indian J Dermatol 2014; 59: 364-368.

Received November 27, 2016. Accepted December 13, 2016. 